\title{
High Energy X-rays for Following Metal-Organic Framework Formation: Identifying Intermediates in Interpenetrated MOF-5 Crystallisation
}

Yue Wu, ${ }^{1}$ Matthew I. Breeze, ${ }^{2}$ Dermot O’Hare ${ }^{1}$ and Richard I. Walton ${ }^{2 *}$

1. Inorganic Chemistry Laboratory, South Parks Road, Oxford, OX1 3QR, U.K.

2. Department of Chemistry, University of Warwick, Coventry, CV4 7AL, U.K.

email: r.i.walton@warwick.ac.uk

\begin{abstract}
We report a study of the solvothermal formation of the interpenetrated form of the zinc 1,4-benzene dicarboxylate (BDC) metal organic framework MOF-5 using high-energy in situ X-ray diffraction $(\lambda=$ $0.2333 \AA, 53.14 \mathrm{keV}$ ). This method allows penetration of a Teflon-lined stainless steel reaction vessel to measure high-resolution powder patterns at $60 \mathrm{~s}$ intervals from which temporal evolution of lattice parameters and Bragg peak area can be refined. Crystallisation in $N, N$-dimethylformamide (DMF) solvent in the presence of melamine at $110{ }^{\circ} \mathrm{C}$ yields interpenetrated MOF-5 upon extended periods of reaction (12 hours). Laboratory quenching experiments show that a structurally unrelated material, $\mathrm{Zn}(\mathrm{BDC})(\mathrm{DMF})$, can be isolated at short reaction times (0.5 - 4 hours), while the in situ experiment reveals the presence of a different phase under reaction conditions at similar times. Profile fitting of the diffraction pattern measured in situ allows an orthorhombic Pcca unit cell ( $a=9.6589$ (2) $\AA, b=$ 18.3096(3) $\AA, c=25.4547(14) \AA$ ) to be refined, providing a tentative identification as a high temperature polymorph of $\mathrm{Zn}_{3}(\mathrm{BDC})_{3}\left(\mathrm{H}_{2} \mathrm{O}\right)_{2} \cdot 4 \mathrm{DMF}$. The phase seen in situ and the one isolated on quenching have no structural relationship to the MOF-5 framework being constructed from different secondary building units and with different topologies. This illustrates the facile interconversion between crystalline metal carboxylate MOFs in reactive solutions.
\end{abstract}

Paper based on a presentation at the International Zeolite Conference, Rio de Janeiro, Brazil, June 2016, for the special issue of Microporous and Mesoporous Materials 


\section{Introduction}

The solvothermal crystallisation of metal organic framework (MOF) materials has yielded a vast number of new porous solids over the past 15 years, some of which are being explored for their properties in practical application for molecular sieving, gas capture and storage and shape-selective heterogeneous catalysis, of relevance in important issues relating to sustainable energy and processing of chemicals [1-4]. Given the large choice of combinations of variables available in MOF crystallisation, from the metals and ligands, to solvent, temperature and reaction time, it would be highly desirable to have some predictive ability so to plan a synthesis to target the formation of a desired material with structure, and hence porosity and reactivity, tuned for a chosen application. Unfortunately, this is presently not the case; indeed, while it may be anticipated that certain ligand geometries coupled with the coordination preferences of particular metal ions might yield framework structures 'by design', the fact is that polymorphism often occurs and effects such as solvent templating are difficult to predict. This means that embarking on the synthesis of new MOF materials is not necessarily straightforward $[5,6]$.

An important approach to furthering knowledge of MOF crystallisation is to perform in situ studies of their formation: by following changes in atomic-scale structure during crystallisation a material's assembly can be tracked and mechanisms for crystal growth deduced [7]. There are several experimental probes appropriate for following solvothermal crystallisation $[8,9]$ many of which are applicable for MOFs: these range from spectroscopic methods that probe local atomic structure, such as NMR [10], and EXAFS [11], to small-angle scattering techniques that follow the formation of particle evolution from the nano- to the micron-scale $[12,13]$, via diffraction methods that detect long-range crystallographic order $[14,15]$. To apply these methods experimental cells must be designed that mimic solvothermal reactors used in the laboratory while permitting time-resolved data to be measured during crystallisation: a compromise must often be reached between the cell design and the data quality [7]. The literature, however, contains some significant examples of these measurements that have allowed crystallisation pathways for a number of prototypical MOFs to be followed: for example the iron carboxylate MIL-53 was observed to form via a structurally unrelated phase MOF-235, by in situ Xray diffraction [14], which was later also shown to be the case for the aluminium analogue with combined WAXS-SAXS (combined wide-angle and small-angle X-ray scattering) [16], and small angle $\mathrm{X}$-ray scattering has been used to follow the formation of zeolite imizadolate frameworks, where formation of amorphous primary particles was detected followed by an attachment of those particles to nuclei for crystal growth [12].

Time-resolved X-ray diffraction (XRD) is a natural method of choice for following crystallisation and although the method can only detect material once crystal domains of a certain size are reached, it is particularly powerful when tracking the successive formation of materials in a solvothermal reaction: 
this has been demonstrated for some diverse families of MOF materials [17-20]. Early in situ diffraction studies used the energy-dispersive method with an energy range of high intensity X-ray radiation to allow penetration of solvothermal autoclaves and fast data collection from a static detector, but with the disadvantage of low $d$-spacing resolution that limits the amount of structural information that can be extracted [14]. We have recently shown that by using monochromatic, high-energy (>50 keV) X-rays we can measure data from a realistic solvothermal reactor $(\sim 5 \mathrm{ml}$ in volume) with subsecond time resolution and angular resolution to permit Rietveld refinement of crystal structure [21]. In this paper we provide a new example of the use of this technique and consider the case of MOF-5, one of the prototypical metal organic frameworks [22]. We here investigate the formation of the interpenetrated form of MOF-5, following the synthesis method of Kim et al. who used melamine as an additive in synthesis to direct the formation of the interpenetrated material [23]. Interpenetrated MOFs, those that contain inter-twinned distinct coordination networks, are topical since control of interpenetration provides a means of tuning porosity in the solid state [24]. We show how the high resolution of the high energy X-ray diffraction data recorded in situ from solids suspended in solvent allow unambiguous phase identification, which in turn reveals that at the early stages of reaction the formation of crystalline precursors occurs before the final MOF material is produced. While our earlier work using this method used polymer or glass reactors to minimise background scatter [20,21], we now also show how high resolution powder data may be recorded from a $20 \mathrm{~mL}$ Teflon-lined steel autoclave, of the type used in preparative laboratory synthesis for many families of inorganic materials.

\section{Experimental Section}

Beamline I12 of the Diamond Light Source was used for in situ diffraction experiments: this receives $\mathrm{X}$-rays in the energy range 53-150 keV and can be used in either monochromatic or energy dispersive modes [25]. We used a fixed incident X-ray wavelength of $0.2333 \AA$ ( $53.14 \mathrm{keV})$, selected using a Bent crystal double Laue geometry monochromator monochromator, with diffraction patterns recorded using a $43 \times 43 \mathrm{~cm}^{2}$ Thales Pixium image plate detector. The detector was calibrated with a crystalline $\mathrm{CeO}_{2}$ reference and the data were integrated using the DAWN software, normalising to the incident beam intensity [26]. Profile analysis of the diffraction patterns was performed using the TOPAS software [27]: this allowed phase identification, refinement of lattice parameters and profile fits using the Pawley method. The Oxford-Diamond in situ cell (ODISC) [28] was used to heat a $20 \mathrm{~mL}$ Teflon-lined steel autoclave with steel walls, of the type previously used for the study of hydrothermal crystallisations by O'Hare and co-workers [29]. The ODISC cell uses an IR furnace to permit rapid heating of the sample environment. For the synthesis of interpenetrated MOF-5 the method of Kim et al. was used [23]. In the laboratory, this was performed in screw-top glass tubes heated in an oil-bath and agitated with a magnetic stirrer: this was done to first mimic the rapid heating of the in situ experiment but also to allow visual observation of the formation of solid material from the initial clear solution. This reaction was scaled three-fold for the in situ X-ray diffraction experiments: $1.8 \mathrm{~g} \mathrm{Zn}\left(\mathrm{NO}_{3}\right)_{3} \cdot 6 \mathrm{H}_{2} \mathrm{O}, 0.375 \mathrm{~g} \mathrm{1,4-}$ 
benzenedicarboxylic acid and $0.15 \mathrm{~g}$ melamine was added to $15 \mathrm{ml}$ DMF in the Teflon liner. The mixture was sealed in the reactor and agitated using a magnetic stirrer for 10 minutes and then the temperature increased to $110{ }^{\circ} \mathrm{C}$ and data collection started immediately.

Laboratory powder XRD was used to identify the materials produced in preliminary experiments: patterns were measured using a D5000 diffractometer operating with $\mathrm{CuK} \alpha 1 / 2$ radiation.

\section{Results and Discussion}

Powder XRD showed the sucessful formation of the interpenetrated form of MOF-5, Figure 1, after an extended reaction time of 20 hours was used. If considerably shorter times of reaction were used the product was a phase-pure sample of $\mathrm{Zn}(\mathrm{BDC})(\mathrm{DMF})$, whose synthesis and structure was previously reported by Hawxwell et al. [30]. This material has a completely different topology than the MOF-5 network, being constructed from a $\mathrm{Zn}_{2}\left(\mathrm{CO}_{2}\right)_{4}(\mathrm{DMF})_{2}$ paddle-wheel secondary building unit connected in two dimensions by bridging BDC linkers to give a square-grid, Figure 2. Interestingly, this intermediate phase is different to that seen by McKinstry et al., who found that by quenching studies of MOF-5 synthesis the material MOF-69c, $\mathrm{Zn}_{3}(\mathrm{OH})_{2}(\mathrm{BDC})_{2}$ forms at short reaction times [31], although in that work $N, N$-diethylformamide was used as solvent, as indeed is more commonly the case for MOF5 , rather than the $N, N$-dimethylformamide that we have used. In the original synthesis of interpenetrated MOF-5, Kim et al. reported that $\mathrm{Zn}(\mathrm{BDC})(\mathrm{DMF})$ formed from the same reagents but at $\mathrm{pH}<3.5$; here we have found that time is also a factor in the formation of this competitive phase at $\mathrm{pH}=4.0$.

In the laboratory the experiments performed in glass reaction tubes revealed the formation of a precipitate during periods of heating between 30 minutes and 4 hours, but beyond this time of heating the solid first formed redissolved and a clear solution returned, and only at this point could the interpenetrated MOF-5 be isolated from the reaction. In order to understand the nature of this intermediate phase, we performed in situ XRD on a crystallisation at $110{ }^{\circ} \mathrm{C}$. Figure 3 shows a contour map of crystallisation with time: this provides complementary information to the laboratory observations with the emergence of a crystalline material at 50 minutes whose Bragg peak intensities increase to 200 minutes, and then showed the onset of decay (the reaction was terminated at this point and data collection ceased). The formation of this solid material would then correspond to the precipitate formed at the early stages of reaction, with rather similar kinetics of formation between the laboratory and in situ experiments.

The intermediate phase could not be matched to the triclinic $\mathrm{Zn}(\mathrm{BDC})(\mathrm{DMF})$ isolated by quenching, nor is the expected interpenetrated MOF-5 product and a search of the literature found no other benzenedicarboxylates of divalent metals as likely candidates, so the diffraction pattern measured in situ was indexed. Tables showing the indexing results are presented in Supporting Information. Figure 4 shows a Pawley fit to the powder diffraction pattern measured in situ at $200 \mathrm{~min}$ using the orthorhomic 
unit cell $a=9.6589(2) \AA, b=18.3096(3) \AA, c=25.4547(14) \AA$ in space group Pcca.. Identification of a material from its unit cell alone is not unambiguous, but this unit cell matches well that of a cobalt(II) 1,4-benzenedicarboxylate that some of us have recent observed in solvothermal solutions [32]. The measured powder pattern also matches very well that of the cobalt phase in the previous work (see Supporting Information). This is related structurally to a second zinc phase reported by Hawxwell et al. $\mathrm{Zn}_{3}(\mathrm{BDC})_{3}\left(\mathrm{H}_{2} \mathrm{O}\right)_{2}$. $4 \mathrm{DMF}$ [30]. The structure of this material is completely different to the $\mathrm{Zn}(\mathrm{BDC})(\mathrm{DMF})$ isolated on quenching, being constructed from trimeric secondary building units, Figure 5. Despite the lack of structural relationship, either in terms of topology or secondary building units, we do note that the interconversion between zinc 1,4-benzenedicarboxylates, including the transformation of dimeric paddlewheels to chains via a zipping and unzipping process has been reported in solution [33], so this suggests that the conversion between various frameworks in this family is facile and may occur during the reactive solutions prior to the formation of the final MOF product.

Having realised a tentative identification of the transient phase formed early in the reaction, we used the powder diffraction patterns to extract lattice parameters and phase fraction as a function of time, Figure 6. The scale factor (total Bragg peak area), Figure 6a, shows more clearly how the transient phase reaches a maximum quantity around 140 minutes before starting to decay. This is consistent with the laboratory observations that the first precipitate formed at around 20 minutes completely redissolves by 240 minutes, bearing in mind the kinetics between the laboratory and in situ experiments may not be identical. The lattice parameters show an evolution during the lifetime of the precursor phase with the $a$ and $c$ cell lengths showing a gradual decrease and the $b$ length showing an increase. Although the changes are small they are real and within the error of the refined parameters. Thermal expansion is is a possible cause of the shift, although calibration experiments with the reaction cell showed how internal temperature is reached within a matter of minutes with the IR heaters in the ODISC cell [28]. We previously observed a similar shift in lattice parameters during the formation of an ytterbium 1,4benzenedicarboxylate using the same setup but within a polymer reaction cell [21]. With the higher quality of diffraction data measured for that system we could relate this to changes in solvent composition during the formation of the material. The data quality is lower in the present investigation, due to the use of the steel reaction cell with greater background, compounded by the more weakly scattering zinc material we have studied, so we are unable to perform full Rietveld refinement of structure. However, a similar model of solvent exchange of water from the hydrated metal salt precursor by the solvent DMF causing a gradual evolution of lattice parameter is equally plausible during the formation of this zinc material. We also note that some fluctuations are seen in the temporal evolution of lattice parameters, particularly apparent for the $a$ parameter for which the relative change is smallest, Figure $6 \mathrm{~b}$. This may be due to the stirring creating inhomogeneous heating, but this effect was not seen in previous work where the smaller volume polymer reaction tubes $(5 \mathrm{~mL})$ were used [21] and hence may indicate a limitation of using the larger volume $(20 \mathrm{~mL})$ steel vessels in this experimental set-up. 


\section{Conclusions}

We have provided new information about the formation pathways in the formation of one of the prototypical MOF materials. Figure 7 summarises the conclusions of the pathways deduced during the crystallisation of the interpenetrated MOF-5. Although quenching experiments can be used to detect the presence of intermediate phases, our work shows how it is vital to make in situ studies under reaction conditions: the phase we have detected under solvothermal conditions is not the same as the material produced on quenching and extracting the solid product. The results illustrate the complexities of MOF crystallisation, with the presence of transient precursor phases meaning that reaction conditions (particularly time) need to be explored carefully in the discovery of new materials. As we have emphasised, diffraction methods detect the presence of long-range crystal order and no information is gained about the local atomic rearrangements taking place during the solution at the early stages of reaction, nor during the transformation of one crystalline phase to another. Given the very different atomic connectivity of the various materials identified, it seems likely that dissolution-recrystallisation occurs to mediate the transformation of one phase to another. It is therefore important to use complementary local atomic probes to understand fully crystallisation 'mechanism'. However, in situ XRD will always be vital to pinpoint crystallisation events and our work shows how even when using Teflon-lined steel autoclaves, high resolution diffraction patterns can be collected using high energy Xrays, from which structural information can be refined.

\section{Acknowledgements}

We thank Diamond Light Source for provision of beamtime and Dr Michael Hart and Dr Thomas Connolley for assistance with running the experiments on Beamline I12, and we are grateful to the EPSRC (EP/I020691) and the EU SHYMAN (FP7-NMP4-LA-2012-280983) project for funding and Diamond Light Source for provision of beamtime. 


\section{Figures}

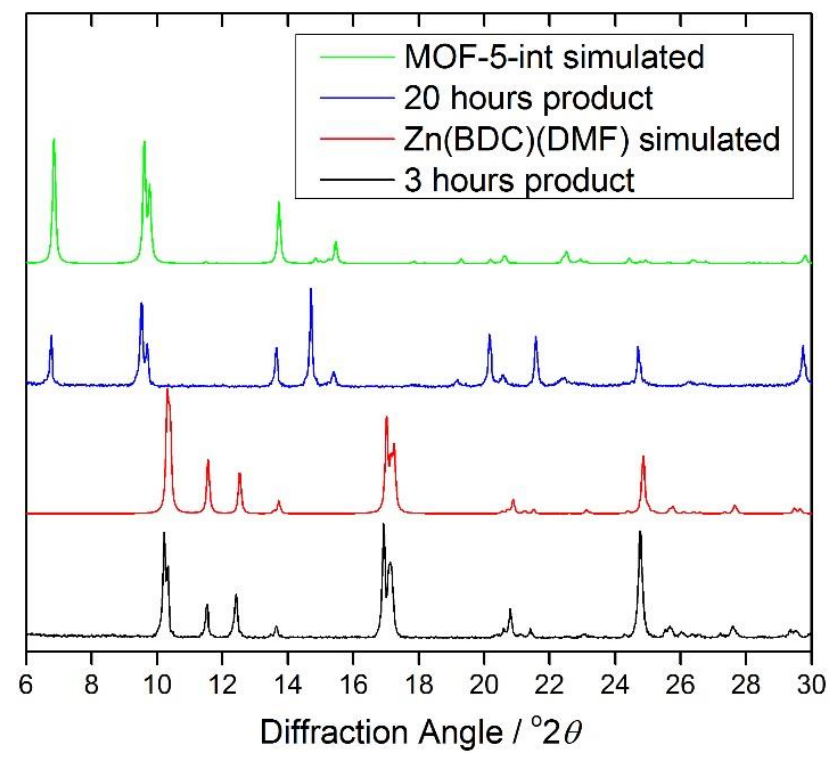

Figure 1: Laboratory powder X-ray diffraction $(\lambda=1.5418 \AA)$ showing the formation of interpenetrated MOF-5 (MOF-5int) and the identification of the precursor phase $\mathrm{Zn}(\mathrm{BDC})(\mathrm{DMF})$ isolated on quenching. The simulated patterns for MOF-5int [23] and $\mathrm{Zn}(\mathrm{BDC})(\mathrm{DMF})$ [30] were generated from published crystal structures. 
(a)

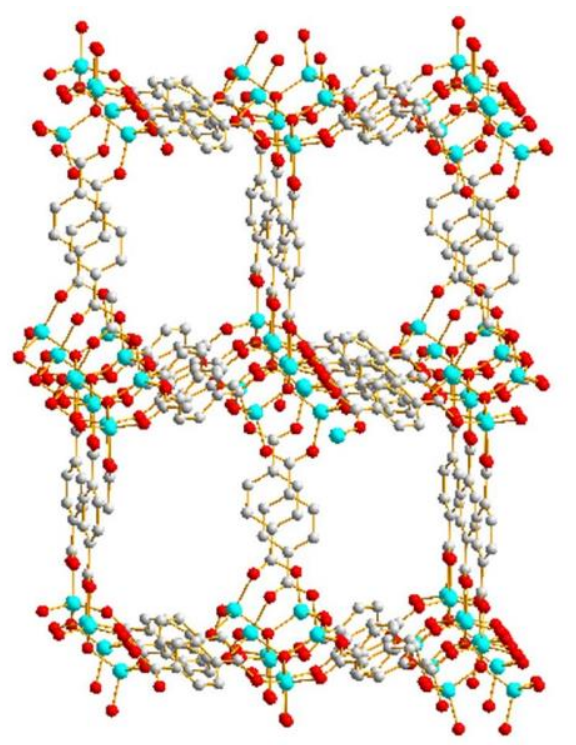

(b)

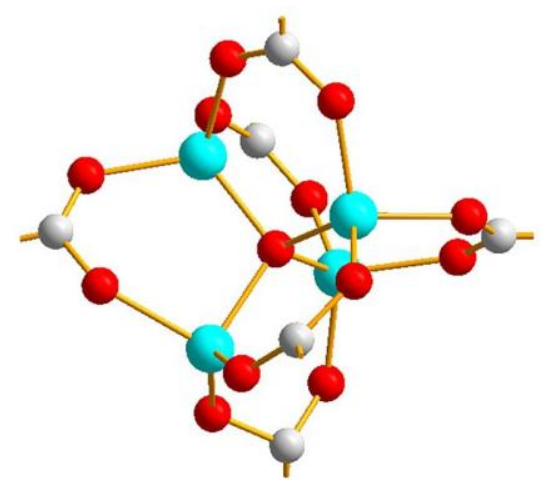

(c)

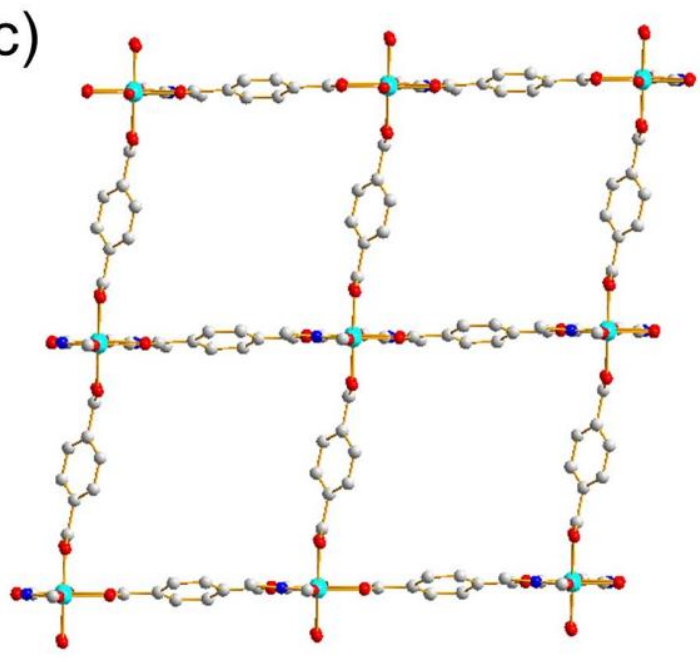

(d)

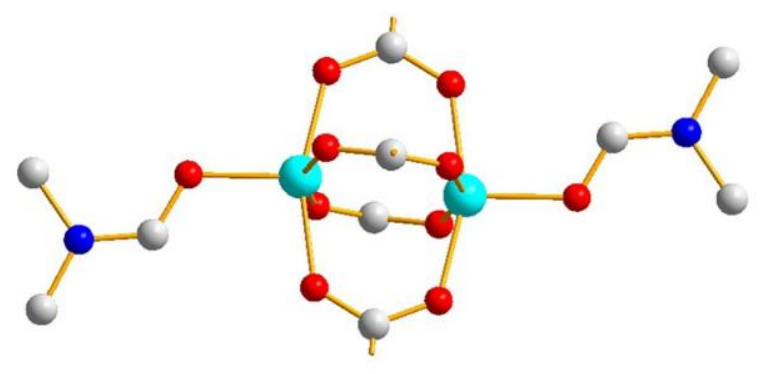

Figure 2: Comparison of the crystal structures of (a) MOF-5int[23] and (c) Zn(BDC)(DMF) [30] drawn using published crystallographic data, with (b) and (d) showing the structures of the respective secondary building units in each. Carbon atoms are pale grey, oxygen atoms read, nitrogen blue and zinc cyan. Incomplete bonds show connections to neighbouring carbon atoms. 


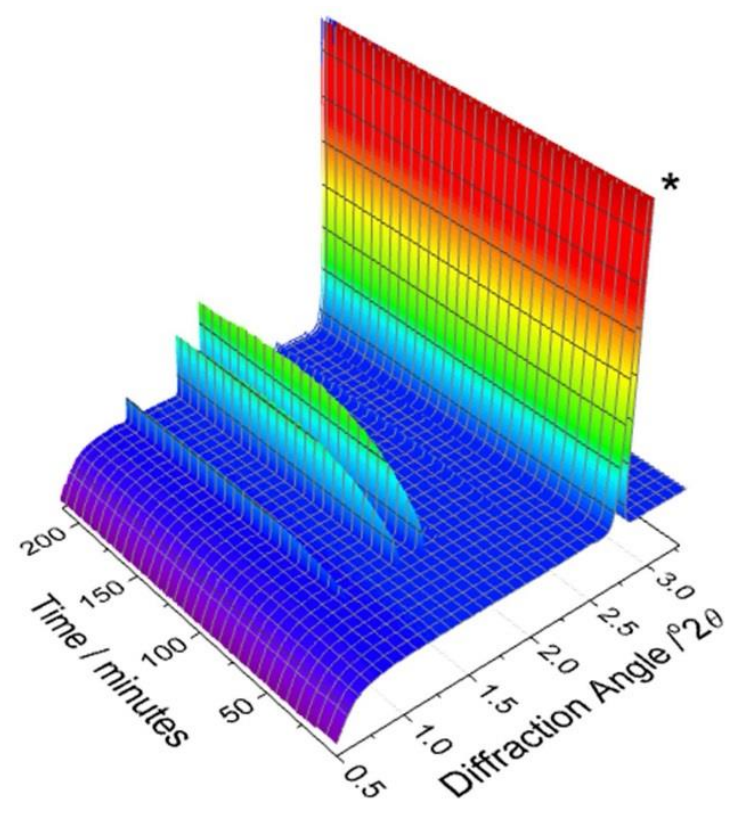

Figure 3: In situ contour map measured during crystallisation at $110{ }^{\circ} \mathrm{C}(\lambda=0.2333 \AA)$. * indicates a Bragg peak due to the steel case of the autoclave.

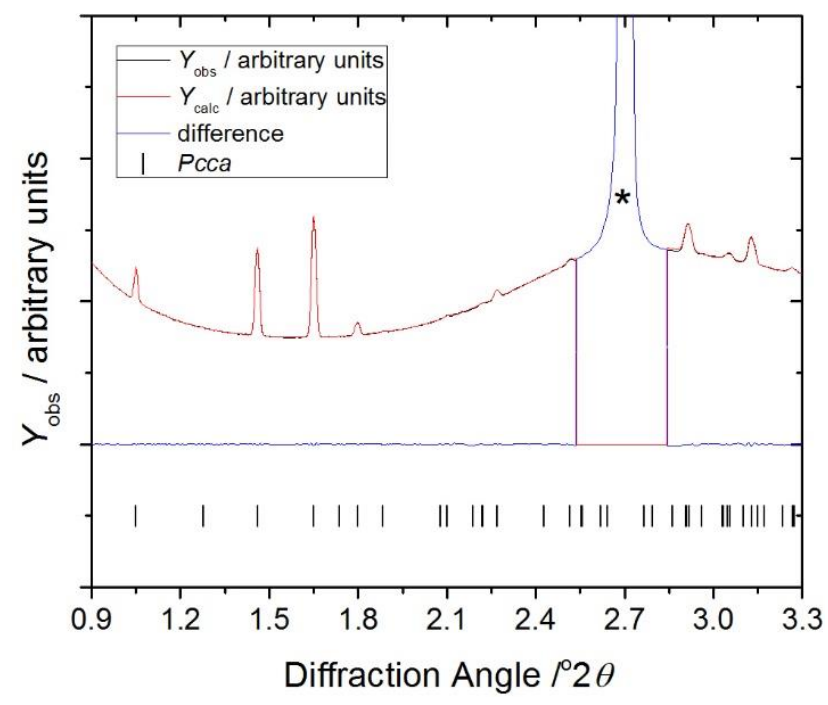

Figure 4: Final profile fit (Pawley method) to the crystalline intermediate phase. The excluded region from 2.55 to $2.85^{\circ}$ is due to the steel autoclave case (indicated by $*$ ). The tick marks are the allowed positions of Bragg peaks for the refined Pcca unit cell $a=9.6589$ (2) $\AA, b=18.3096$ (3) $\AA, c=$ 25.4547(14) $\mathrm{A}$. 

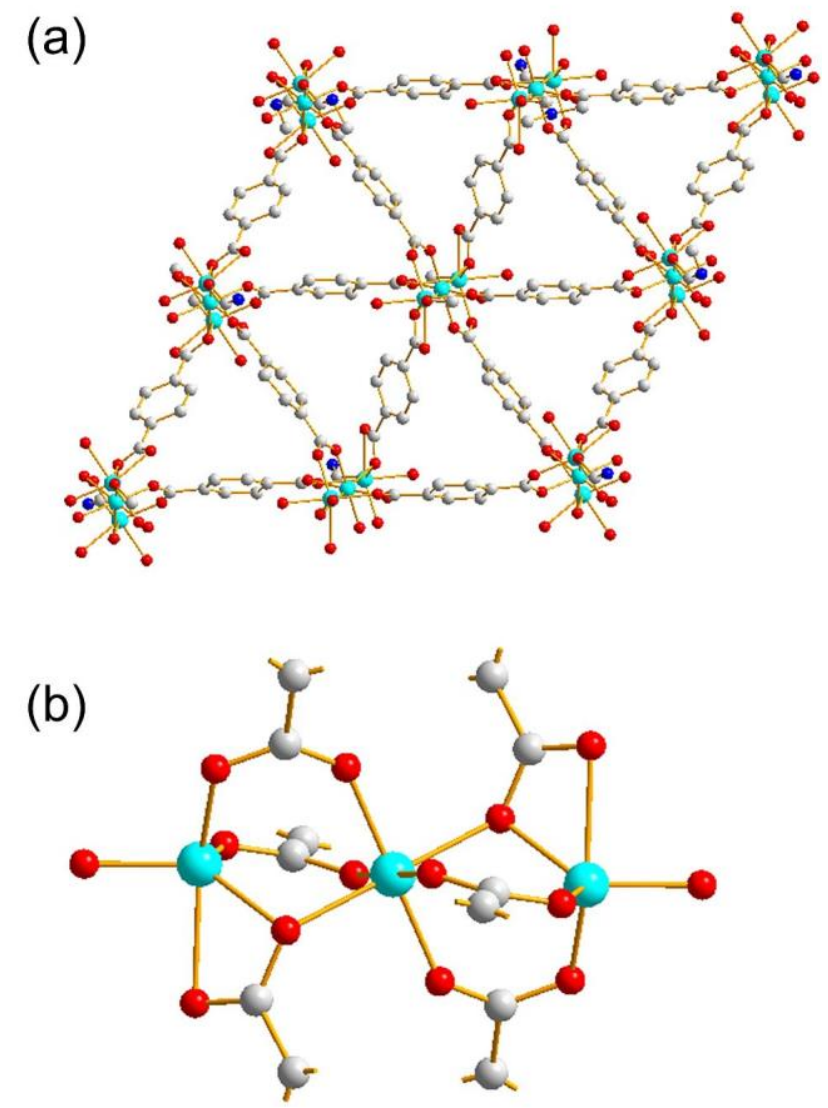

Figure 5: Views of the structure of $\mathrm{Zn}_{3}(\mathrm{BDC})_{3}\left(\mathrm{H}_{2} \mathrm{O}\right)_{2} \cdot 4 \mathrm{DMF}$ drawn using the crystal structure data of Hawxwell et al. [30] and a candidate structure for the intermediate phase seen by in situ XRD: (a) view of the one part of the layered structure and (b) view of the trimeric secondary building unit.
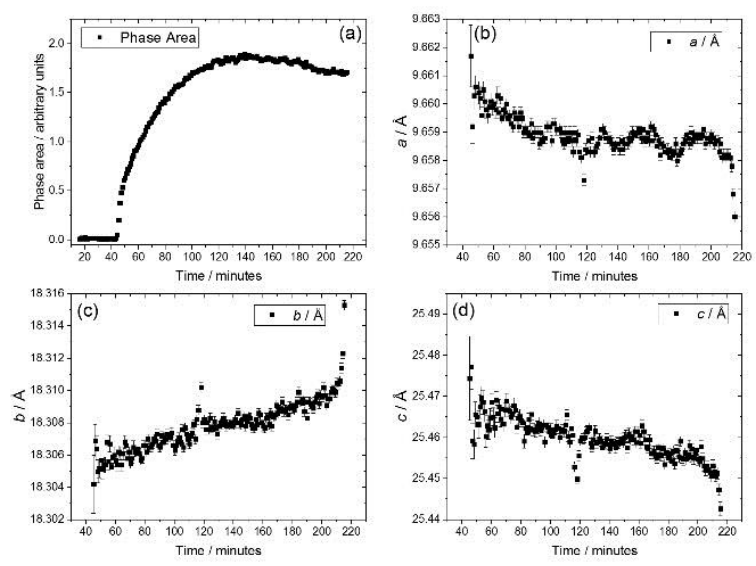

Figure 6: Evolution of (a) Bragg peak area (scale factor) and (b-d) lattice parameters of the precursor phase seen as determined by sequential fitting of in situ XRD patterns during solvothermal synthesis. 


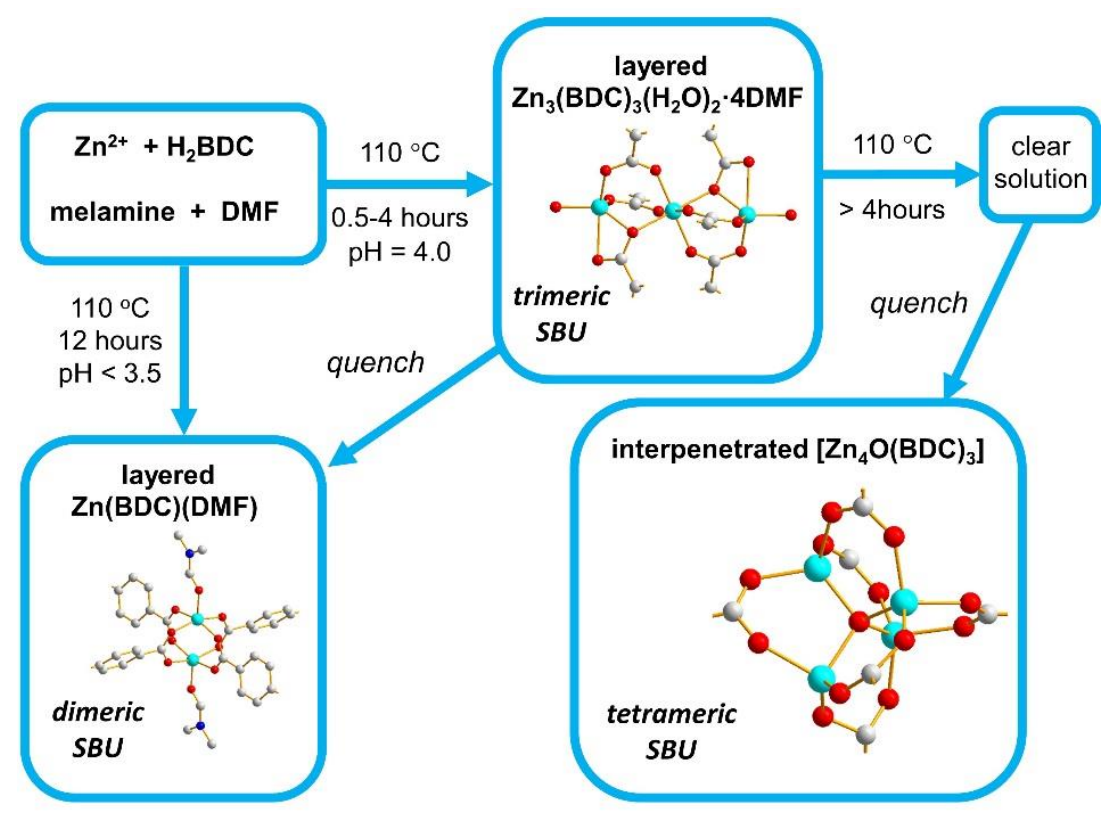

Figure 7: Schematic of reaction pathways leading to the formation of interpenetrated MOF-5 based on results from this work and observations reported by Kim et al. [23]

\section{References}

[1] M. Schröder (Ed.), Functional Metal-Organic Frameworks: Gas Storage, Separation and Catalysis, Springer-Verlag Berlin Heidelberg, 2010.

[2] D. Farrusseng (Ed.), Metal-Organic Frameworks: Applications from Catalysis to Gas Storage, Wiley-VCH Verlag, Weinheim, 2011.

[3] H. Furukawa, K.E. Cordova, M. O'Keeffe, O.M. Yaghi, Science, 341 (2013) 1230444.

[4] S. Kaskel (Ed.), The Chemistry of Metal-Organic Frameworks: Synthesis, Characterization, and Applications, Wiley-VCH Verlag, Weinheim, 2016

[5] R.E. Morris, ChemPhysChem, 10 (2009) 327-329.

[6] M.G. Goesten, F. Kapteijn, J. Gascon, CrystEngComm, 15 (2013) 9249-9257.

[7] R.I. Walton, F. Millange, in: S. Kaskel (Ed.) The Chemistry of Metal-Organic Frameworks: Synthesis, Characterization, and Applications, Wiley-VCH Verlag, Weinheim, 2016, pp. 729764.

[8] N. Pienack, W. Bensch, Angew. Chem., Int. Edit., 50 (2011) 2014-2034.

[9] K.M.Ø. Jensen, C. Tyrsted, M. Bremholm, B.B. Iversen, ChemSusChem, 7 (2014) 15941611.

[10] G. Férey, M. Haouas, T. Loiseau, F. Taulelle, Chem. Mater., 26 (2014) 299-309.

[11] S. Surblé, F. Millange, C. Serre, G. Férey, R.I. Walton, Chem. Commun,, (2006) 15181520.

[12] J. Cravillon, C.A. Schroder, R. Nayuk, J. Gummel, K. Huber, M. Wiebcke, Angew. Chem., Int. Edit., 50 (2011) 8067-8071.

[13] M.G. Goesten, E. Stavitski, J. Juan-Alcaniz, A. Martinez-Joaristi, A.V. Petukhov, F. Kapteijn, J. Gascon, Catal. Today, 205 (2013) 120-127.

[14] F. Millange, M.E. Medina, N. Guillou, G. Férey, K.M. Golden, R.I. Walton, Angew. Chem., Int. Edit., 49 (2010) 763-766. 
[15] R. El Osta, M. Feyand, N. Stock, F. Millange, R.I. Walton, Powder Diffr., 28 (2013) S256S275.

[16] E. Stavitski, M. Goesten, J. Juan-Alcaniz, A. Martinez-Joaristi, P. Serra-Crespo, A.V. Petukhov, J. Gascon, F. Kapteijn, Angew. Chem., Int. Edit., 50 (2011) 9624-9628.

[17] F. Millange, R. El Osta, M.E. Medina, R.I. Walton, CrystEngComm, 13 (2011) 103-108.

[18] M. Feyand, A. Hubner, A. Rothkirch, D.S. Wragg, N. Stock, Inorg. Chem., 51 (2012) 12540-12547.

[19] Y. Wu, S.J. Moorhouse, D. O'Hare, Chem. Mat., 27 (2015) 7236-7239.

[20] H.H.M. Yeung, Y. Wu, S. Henke, A.K. Cheetham, D. O'Hare, R.I. Walton, Angew. Chem., Int. Edit., 55 (2016) 2012-2016.

[21] Y. Wu, M.I. Breeze, G.J. Clarkson, F. Millange, D. O'Hare, R.I. Walton, Angew. Chem., Int. Edit., 55 (2016) 4992-4996.

[22] N.L. Rosi, J. Eckert, M. Eddaoudi, D.T. Vodak, J. Kim, M. O'Keeffe, O.M. Yaghi, Science, 300 (2003) 1127-1129.

[23] H. Kim, S. Das, M.G. Kim, D.N. Dybtsev, Y. Kim, K. Kim, Inorg. Chem., 50 (2011) 36913696.

[24] H.L. Jiang, T.A. Makal, H.C. Zhou, Coord. Chem. Rev., 257 (2013) 2232-2249.

[25] M. Drakopoulos, T. Connolley, C. Reinhard, R. Atwood, O. Magdysyuk, N. Vo, M. Hart, L. Connor, B. Humphreys, G. Howell, S. Davies, T. Hill, G. Wilkin, U. Pedersen, A. Foster, N. De Maio, M. Basham, F. Yuan, K. Wanelik, J. Synchrotron Radiat., 22 (2015) 828-838.

[26] M. Basham, J. Filik, M.T. Wharmby, P.C.Y. Chang, B. El Kassaby, M. Gerring, J. Aishima, K. Levik, B.C.A. Pulford, I. Sikharulidze, D. Sneddon, M. Webber, S.S. Dhesi, F. Maccherozzi, O. Svensson, S. Brockhauser, G. Naray, A.W. Ashton, J. Synchrotron Radiat., 22 (2015) 853-858.

[27] A. Coelho, TOPAS-Academic, Coelho Software, Brisbane, Australia

[28] S.J. Moorhouse, N. Vranjes, A. Jupe, M. Drakopoulos, D. O'Hare, Rev. Sci. Instrum., 83 (2012) 084101

[29] J.S.O. Evans, R.J. Francis, D. O'Hare, S.J. Price, S.M. Clark, J. Flaherty, J. Gordon, A. Nield, C.C. Tang, Rev. Sci. Instrum., 66 (1995) 2442-2445.

[30] S.M. Hawxwell, H. Adams, L. Brammer, Acta Crystallogr. Sect. B, 62 (2006) 808-814. [31] C. McKinstry, E.J. Cussen, A.J. Fletcher, S.V. Patwardhan, J. Sefcik, Crystal Growth \& Design, 13 (2013) 5481-5486.

[32] Y. Wu, S. Henke, G. Kieslich, I. Schwedler, M. Yang, D.A.X. Fraser, D. O'Hare, Angew. Chem., Int. Ed. 55 (2016) 14081-14084

[33] P. Smart, C.A. Mason, J.R. Loader, A. Meijer, A.J. Florence, K. Shankland, A.J. Fletcher, S.P. Thompson, M. Brunelli, A.H. Hill, L. Brammer, Chem. Eur. J., 19 (2013) 3552-3557. 